\title{
Hydrogen Molecules Rotational Stimulated Raman Scattering in All-Fiber Cavity Based on Hollow-Core Photonic Crystal Fibers
}

\author{
Wenxi Pei ${ }^{1,2}$, Hao Li ${ }^{1,2}$, Wei Huang ${ }^{1,3}$, Meng Wang ${ }^{1,2,3}$ and Zefeng Wang 1,2,3,* \\ 1 College of Advanced Interdisciplinary Studies, National University of Defense Technology, \\ Changsha 410073, China; peiwenxi@nudt.edu.cn (W.P.); lihao18c@nudt.edu.cn (H.L.); \\ cuiyulong@nudt.edu.cn (W.H.); wangmeng@nudt.edu.cn (M.W.) \\ 2 Hunan Provincial Key Laboratory of High Energy Laser Technology, Changsha 410073, China \\ 3 State Key Laboratory of Pulsed Power Laser Technology, Changsha 410073, China \\ * Correspondence: zefengwang@nudt.edu.cn
}

Citation: Pei, W.; Li, H.; Huang, W.; Wang, M.; Wang, Z. Hydrogen

Molecules Rotational Stimulated

Raman Scattering in All-Fiber Cavity Based on Hollow-Core Photonic

Crystal Fibers. Crystals 2021, 11, 711.

https://doi.org/10.3390/cryst11060711

Academic Editors: David Novoa and Nicolas Y. Joly

Received: 22 May 2021

Accepted: 18 June 2021

Published: 21 June 2021

Publisher's Note: MDPI stays neutral with regard to jurisdictional claims in published maps and institutional affiliations.

Copyright: (c) 2021 by the authors. Licensee MDPI, Basel, Switzerland. This article is an open access article distributed under the terms and conditions of the Creative Commons Attribution (CC BY) license (https:/ / creativecommons.org/licenses/by/ $4.0 /)$.

\begin{abstract}
Here, we report the rotational stimulated Raman scattering (SRS) of hydrogen molecules in an all-fiber cavity based on hollow-core photonic crystal fibers (HC-PCFs). The gas cavity consists of a $49 \mathrm{~m}$ long HC-PCF filled with 18 bar high-pressure hydrogen and two sections of fusion spliced solid-core fibers on both ends. When pumped by a homemade $1064 \mathrm{~nm}$ pulsed fiber amplifier, only rotational SRS occurs in the gas cavity due to the transmission spectral characteristics of the used HC-PCF, and $1135 \mathrm{~nm}$ Stokes wave is obtained (Raman frequency shift of $587 \mathrm{~cm}^{-1}$ ). By changing the pulse width and repetition frequency of the pump source, the output characteristics are explored. In addition, a theoretical model is established for comparison with the experimental results. This work is helpful for the application of gas Raman laser based on the HC-PCFs.
\end{abstract}

Keywords: photonic crystal fiber; hollow-core fiber; fiber gas laser; stimulated Raman scattering

\section{Introduction}

Since the first demonstration in 1963 [1], stimulated Raman scattering (SRS) in gases has been considered as an effective way of generating lasers at unobtainable wavelengths. Due to the large Raman shift and high Raman gain coefficient, light sources based on the SRS in gases can obtain tunable, narrow linewidth lasers, especially for the lasers in the ultraviolet and infrared spectral range [2,3]. However, the SRS in the gas cell has a short interaction length, which requires very high pump peak power, usually up to megawatt level. Besides, there are always more than one Raman line generated and the Raman conversion efficiency is quite low for the lasers at the desired wavelength. The appearance of hollow-core fibers ( $\mathrm{HCFs}$ ) provides an effective solution to these problems and gives birth to a new kind of fiber lasers, namely, fibers gas Raman lasers (FGRLs). The gas cell based on HCFs can greatly enhance the interaction between the pump laser and the gas molecules. The interaction length can be also greatly extended by increasing the length of the HCFs. What is more, the final gain spectrum of various orders of Stokes waves can be controlled by wavelength-dependent fiber attenuation in the HCFs, which can provide much higher conversion efficiency of the desired wavelength. Gaseous hydrogen is the most commonly used Raman gain medium in gas cells. The first demonstration of the SRS of hydrogen molecules in HCFs was in 2002 [4], then a lot of work has been done based on various HCFs [4-15]. However, in almost all reported FGRLs, the pump laser is coupled into the HCFs through free space, which is quite inconvenient and limits the applications of the FGRLs. Thus, in 2004 [13], Benabid et al. first reported an all-fiber gas cell spliced by an expensive special fiber fusion splicer (Vytran FFS-2000-PM, Morganville, NJ, USA), which is an effective but not common way. In 2007 [6], Couny et al. reported an all-fiber gas cavity, but the pump laser was coupled into the cavity through free space. Therefore, they did 
not realize the experimental system with all-fiber structure. In our previous work [11,15], we reported the $1.7 \mu \mathrm{m}$ quasi all-fiber FGRL, and the optical isolators were introduced to avoid Fresnel reflection damage to the pump source. The fiber laser was used as the pump source and the pigtail was spliced to the input end of the HC-PCF, which greatly improves the stability of the system. However, the output end of the HC-PCF is still sealed in the gas chamber, making the experimental system still bulky. Chen et al. reported a $1135 \mathrm{~nm}$ FGRL with all-fiber structure in 2013 [14], but the power is less than $1 \mathrm{~mW}$ and the conversion efficiency is only $2.69 \%$, meaning poor performance, and it is quite difficult to explore the applications.

In this paper, we report the hydrogen SRS in an all-fiber cavity based on HC-PCFs. The gas cavity is fabricated by splicing the HC-PCFs with two sections of solid-core fibers at each end using arc fusion technique [16-18]. When pumped with a pulsed $1064 \mathrm{~nm}$ fiber laser amplifier, a $1135 \mathrm{~nm}$ Stokes wave is generated by pure rotational SRS of hydrogen molecules due to the transmission loss spectrum of the HC-PCF. The output characteristics are explored with different pump pulse widths and repetition frequencies. When the width is $4 \mathrm{~ns}$ and the repetition frequency is $20 \mathrm{MHz}$, the maximum Stokes power of $289 \mathrm{~mW}$ is achieved with a power conversion efficiency of $28.5 \%$, which can be greatly enhanced by improving the splicing loss and optimizing the HCF's length in the future. A theoretical model is established, and the experimental result is in good agreement with the simulation result.

\section{Experimental Setup}

Figure 1 shows the experimental setup of pulsed $1 \mu \mathrm{m}$ all-fiber Raman laser. The pump source consists of a seed which is a $1064 \mathrm{~nm}$ optical pulse machine (OPM; a laser diode with adjustable pulse generator, pulse driver, and efficient TEC controller) with multi-longitudinal modes and a homemade double-cladding ytterbium-doped fiber amplifier (YDFA). The pulse width of the seed can be adjusted form $1 \mathrm{~ns}$ to $6 \mathrm{~ns}$ and the repetition frequency tuning range is from $4 \mathrm{kHz}$ to $25 \mathrm{MHz}$. The maximum average output power of $52.42 \mathrm{~mW}$ can be achieved when the seed is at the pulse width of $4 \mathrm{~ns}$ and the repetition frequency is $20 \mathrm{MHz}$. Figure 2a presents the measured fine spectrum with an OSA resolution of $0.02 \mathrm{~nm}$ in this power, and it can be seen that there are four longitudinal modes. In YDFA, there are $5 \mathrm{~m}$ ytterbium-doped fibers (YDF; Nufern double clad optical fiber, USA) with the absorption coefficient of $4.95 \mathrm{~dB} / \mathrm{m}$ at $976 \mathrm{~nm}$ and the core diameter is $10 \mu \mathrm{m}$. The YDF is pumped by a $976 \mathrm{~nm}$ laser diode (LD) with the maximum output power of $3.28 \mathrm{~W}$, which is coupled into the YDF by the fiber combiner. YDFA is fused with an optical isolator (the insertion loss is $0.46 \mathrm{~dB}$, the isolation is $38.5 \mathrm{~dB}$ ) to avoid the disturbance caused by the backward laser. The maximum output power of $1.51 \mathrm{~W}$ is achieved with the $3.28 \mathrm{~W}$ pump power at $976 \mathrm{~nm}$ when the pulse width of seed is $4 \mathrm{~ns}$, and the repetition frequency is $20 \mathrm{MHz}$. The measured fine spectrum of the YDFA in Figure $2 \mathrm{~b}$ keeps the characteristics well, and the corresponding wavelengths are $1063.94 \mathrm{~nm}, 1064.38 \mathrm{~nm}, 1064.83 \mathrm{~nm}$, and $1065.27 \mathrm{~nm}$, respectively. The output fiber of the isolator is fused with a fiber coupler (the measured coupling ratio is 99.83:0.17) to monitor the output power of the pump source in real time. The main output fiber of the coupler is fused with the all-fiber gas cavity. A convex-plane lens is placed at the output end of the gas cavity to collimate the laser. Two silver mirrors can help monitor the output spectrum. Besides, the residual pump laser and Stokes laser are separated by adjusting the flippable long-pass filters, which is helpful to measure the power.

For the gas cavity, a $49 \mathrm{~m}$ long HC-PCF (NKT Photonics HC-1060-02, Rudersdal Municipality, Denmark) filled with 18 bar hydrogen is spliced to a solid-core fiber (Corning HI1060, Corning, NY, USA) as the pump injection end and the other end of the HC-PCF is spliced to another solid-core fiber (SM-GDF-10/125-15FA) as the output end. In our work, the end as the pump injection is spliced first and the other end of the HC-PCF is sealed into a specially designed gas chamber, which can keep the HC-PCF vacuumed and fill it with hydrogen. After standing for $10 \mathrm{~h}$, the HC-PCF is taken out from the gas chamber 
and spliced with the solid-core fiber quickly. By calculating the amount of gas leakage in the fusion splicing process [16], the gas pressure in the all-fiber gas cavity is estimated to be 18 bar. Besides, the fusion loss of mode field mismatch can be reduced by using these different solid-core fibers, and the fusion losses of splice 1 and splice 2 is $1.56 \mathrm{~dB}$ and 0.45 $\mathrm{dB}$, respectively. Figure 3 a presents the cross-section of the HC-PCF under the electron microscope (the core diameter is $10 \mu \mathrm{m}$ ), and Figure $3 \mathrm{~b}$ presents the transmission loss and dispersion curves (from the product manual provided by NKT Photonics). It can be seen that the losses at $1064 \mathrm{~nm}$ and $1135 \mathrm{~nm}$ are $\sim 0.083 \mathrm{~dB} / \mathrm{m}$ and $\sim 0.012 \mathrm{~dB} / \mathrm{m}$, respectively.

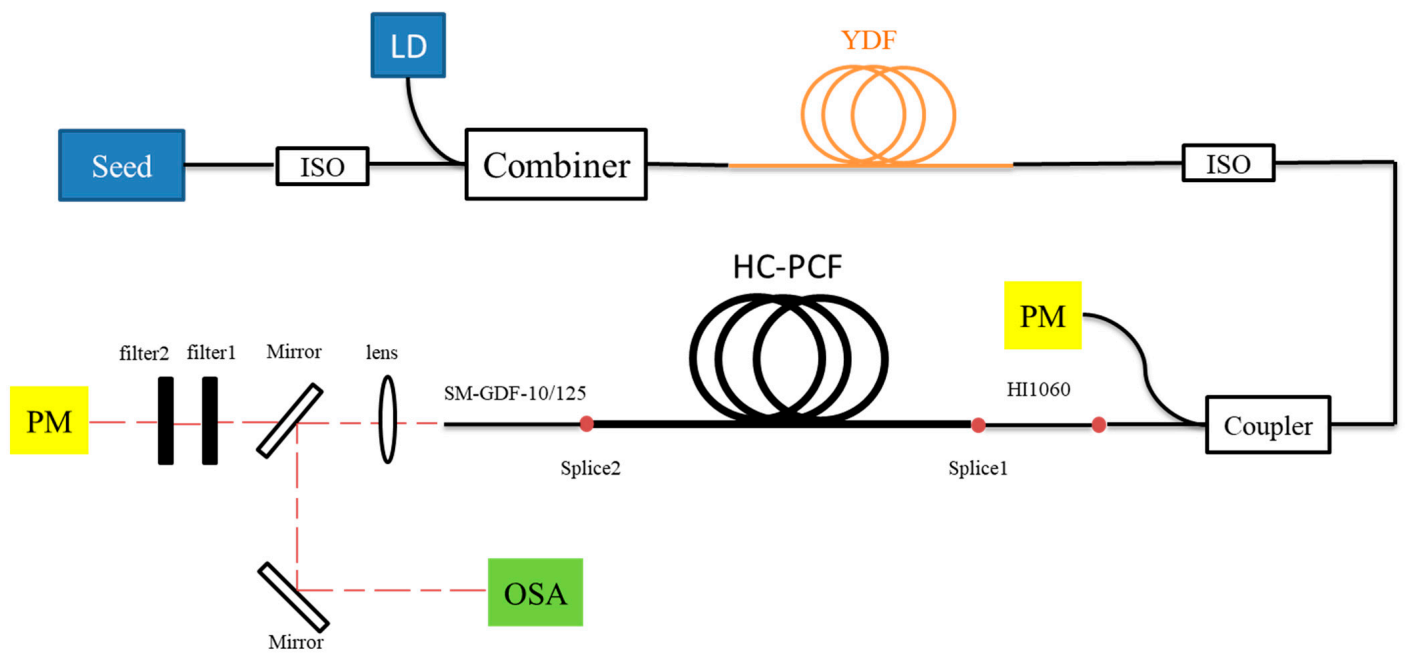

Figure 1. Experimental setup: ISO, optical isolator, LD, laser dioxide, PM, power meter, OSA, optical spectrum analyzer.
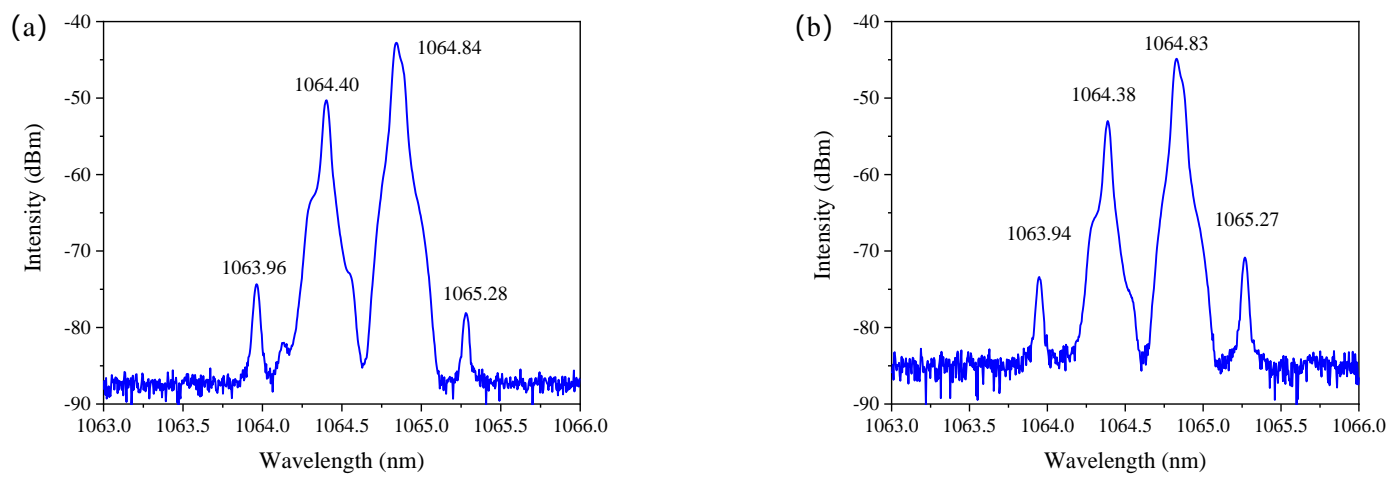

Figure 2. The fine spectrum of (a) the seed and (b) the fiber amplifier at the maximum output power with an OSA resolution of $0.02 \mathrm{~nm}$.

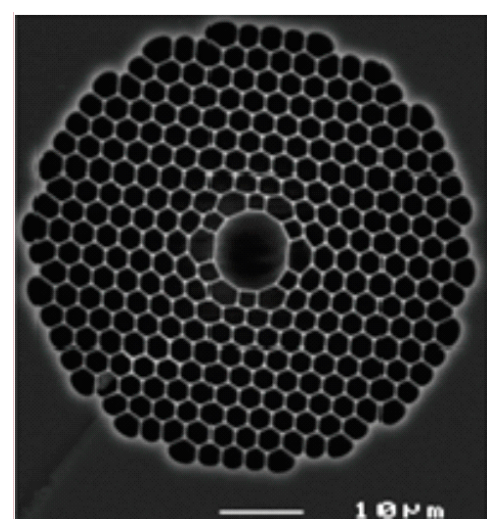

(a)

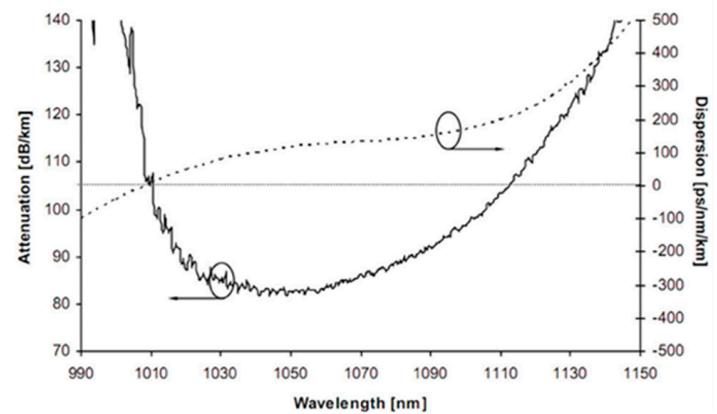

(b)

Figure 3. (a) The schematic cross section of the HC-PCF and (b) the transmission loss and dispersion curves of the HC-PCF, both from the product manual provided by NKT Photonics. 


\section{Experimental Results and Discussion}

The output spectrum is measured when the pump source is at the maximum output power of $1.51 \mathrm{~W}$, as shown in Figure 4a. The width of the pump pulse is $4 \mathrm{~ns}$ and the repetition frequency is $20 \mathrm{MHz}$. It can be seen that besides the pump line at $1064 \mathrm{~nm}$, there are two lines in the spectrum. The line at $1135 \mathrm{~nm}$ is the first-order Raman line, and it is converted from $1064 \mathrm{~nm}$ pump line by the rotational SRS of hydrogen molecules in the gas cavity (the corresponding Raman frequency shift is $587 \mathrm{~cm}^{-1}$ ). The line at $1217 \mathrm{~nm}$ is the second-order Raman line converted from $1135 \mathrm{~nm}$ first-order Raman line with the same Raman frequency shift. Figure $4 \mathrm{~b}-\mathrm{d}$ presents the fine output spectrum of the residual pump laser, the first-order Stokes wave, and the second-order Stokes wave, respectively. The spectrum of the residual pump laser is similar to the output spectrum of the pump source in Figure $2 \mathrm{~b}$, but the intensity of some wavelengths has changed. The lines at $1064.38 \mathrm{~nm}$ and $1064.83 \mathrm{~nm}$ become weaker as they are strong enough to be converted into the first-order Stokes waves; it can be well verified from Figure 4c. There are two lines at $1135.32 \mathrm{~nm}$ and $1135.86 \mathrm{~nm}$ in the spectrum, which represent two different longitudinal modes. They are generated by the rotational SRS of hydrogen molecules pumped by the lasers at $1064.38 \mathrm{~nm}$ and $1064.83 \mathrm{~nm}$, respectively. For the second-order Stokes wave at $1217 \mathrm{~nm}$, there is only one Raman line in the spectrum as shown in Figure $4 \mathrm{~d}$. It is converted by the first-order Stokes wave of $1135.86 \mathrm{~nm}$ as it is strong enough.
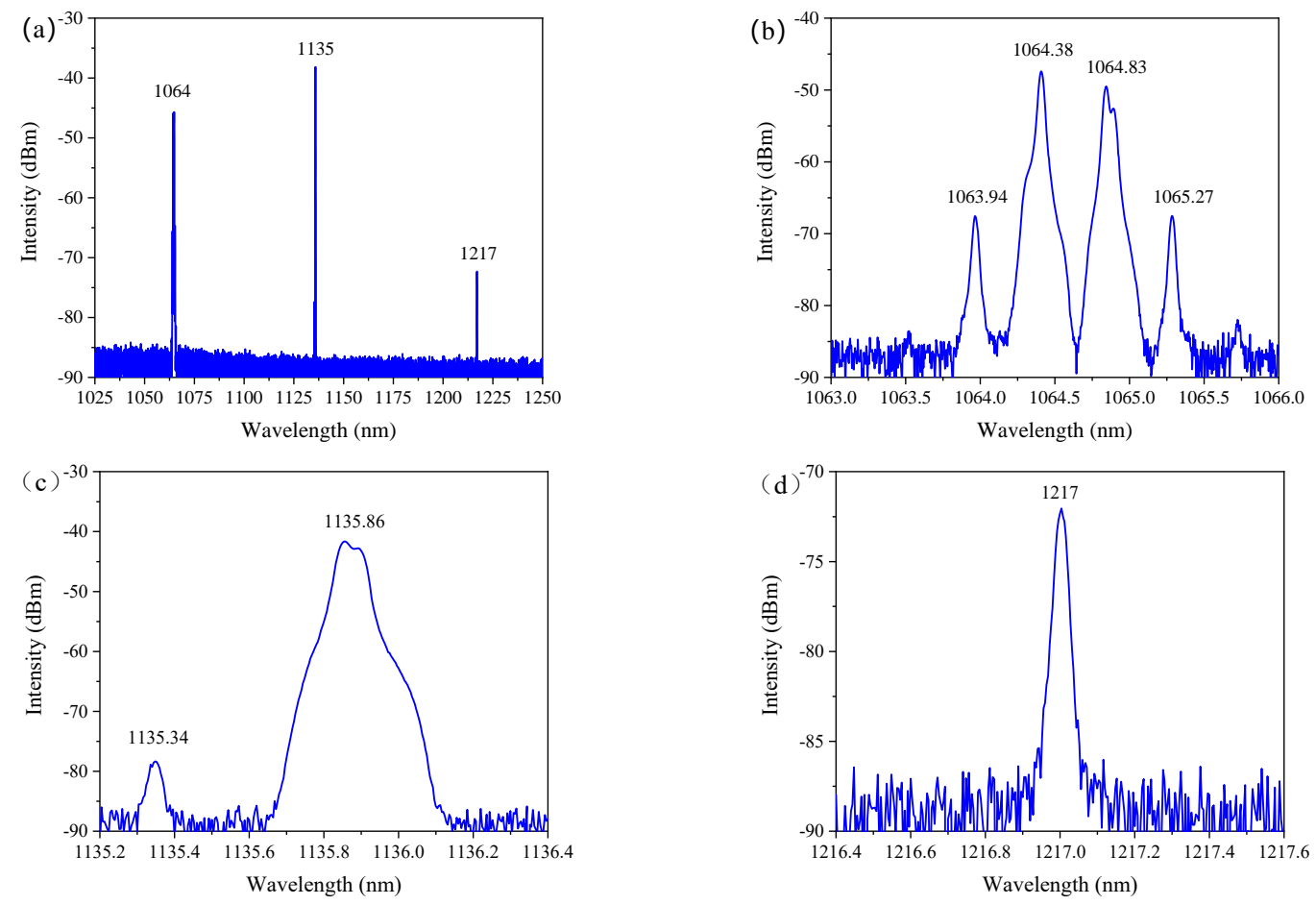

Figure 4. (a) The total output spectrum of $1 \mu \mathrm{m}$ all-fiber FGRL. (b-d) The fine spectrum of the residual pump laser, the first-order Stokes laser, and the second-order Stokes laser, respectively.

To explore the impact of the pulse width on the output characteristics of the all-fiber FGRL, we change the pump pulse width from $4 \mathrm{~ns}$ to $2 \mathrm{~ns}$ and keep the repetition frequency at $20 \mathrm{MHz}$. Figure 5a shows the output spectrum of the pump source with the maximum output power. It can be seen that there are many longitudinal modes. Figure $5 \mathrm{~b}$ presents the output spectrum of the FGRL. There are mainly two lines in the output spectrum: a $1064 \mathrm{~nm}$ pump line and $1135 \mathrm{~nm}$ Raman line. Therefore, only the first-order Stokes wave is generated by the rotational SRS of hydrogen molecules, which is quite different from the case when the pump pulse width is $4 \mathrm{~ns}$. There is a very weak line at $1106 \mathrm{~nm}$ that can only be detected by the highly sensitive OSA. It is also the first-order Stokes wave converted by the $1064 \mathrm{~nm}$ pump laser, and the corresponding Raman frequency shift is 
$354 \mathrm{~cm}^{-1}$. The corresponding Raman gain of $1106 \mathrm{~nm}$ is weaker than that in $1135 \mathrm{~nm}$ [19], so the pump laser is converted into a $1135 \mathrm{~nm}$ Stokes wave first, and only a few pump lasers are converted into $1106 \mathrm{~nm}$ Stokes waves. Figure $5 c$,d shows the fine spectrum of the first-order Stokes waves at $1135 \mathrm{~nm}$ and $1106 \mathrm{~nm}$, respectively, which indicates that only the pump lasers at $1064.38 \mathrm{~nm}$ and $1063.94 \mathrm{~nm}$ are converted into the first-order Stokes waves (the Stokes wave at $1106.1 \mathrm{~nm}$ is converted by the pump laser at $1064.38 \mathrm{~nm}$ ). Due to the accuracy of spectrometer, the linewidth of these lasers cannot be measured accurately, and some Stokes waves are too weak to be measured. Compared with the pump source with the pulse width of $4 \mathrm{~ns}$, there are more longitudinal modes in the pump source when the pulse width is $2 \mathrm{~ns}$, which may lead to the dispersion of power and the Raman conversion is not as sufficient as the case with the pulse width of $4 \mathrm{~ns}$ when the pump power is the same. The absence of the second-order Stokes wave indicates that the first-order Stokes wave is not strong, which also means the conversion is not sufficient.

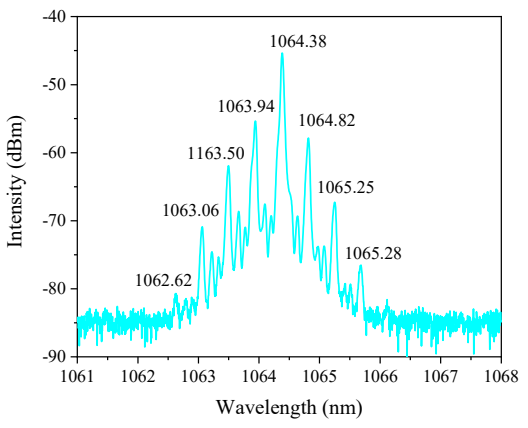

(a)

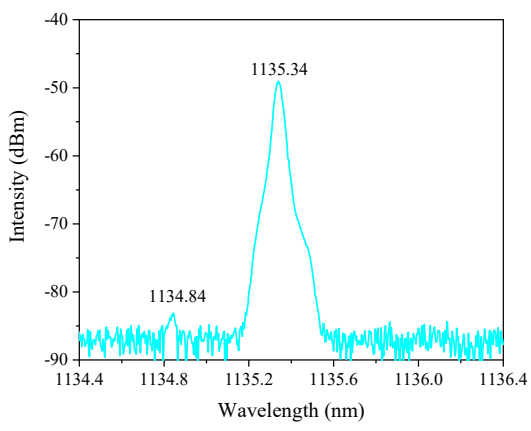

(c)

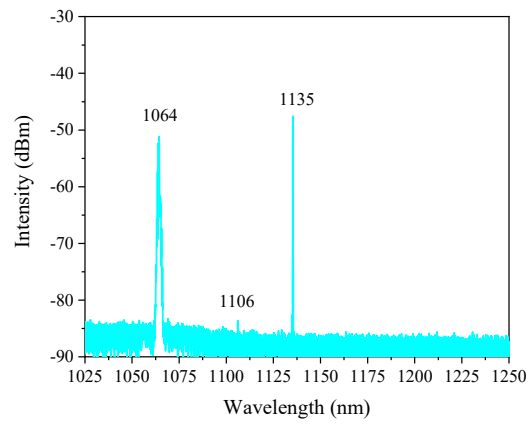

(b)

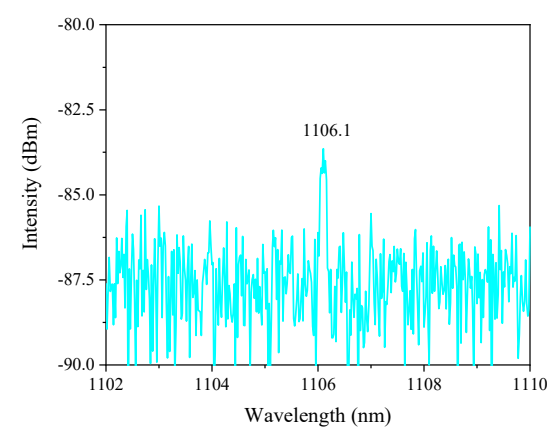

(d)

Figure 5. (a) The output spectrum of the pump source at the maximum output power when the pulse width of the pump laser is $2 \mathrm{~ns}$ and the repetition frequency is $20 \mathrm{MHz}$. (b) The total output spectrum of $1 \mu \mathrm{m}$ all-fiber FGRL in this case. (c,d) The fine spectrum of the first-order Stokes waves at $1135 \mathrm{~nm}$ and $1106 \mathrm{~nm}$, respectively.

To display more clearly the power components of longitudinal modes, the spectral intensity in Figures $2 b$ and $5 a$ is represented by a linear scale, as shown in Figure $6 a, b$. It can be seen that although the output spectrum contains many longitudinal modes when the pump pulse width is $2 \mathrm{~ns}$, the power spectrum mainly contains four components with different wavelengths. The pump lines at $1064.38 \mathrm{~nm}$ and $1063.94 \mathrm{~nm}$ take up the dominant position with the ratio of $77.85 \%$ of the total output power. The power spectrum in Figure $6 \mathrm{~b}$ indicates that when the pulse is $4 \mathrm{~ns}$, the output power has only two main pump lines, and both can be converted into Stokes waves. By calculation, the lines at $1064.83 \mathrm{~nm}$ and $1064.38 \mathrm{~nm}$ account for $90.03 \%$ of the total output power, which is much higher than the case with the pulse width of $2 \mathrm{~ns}$. Therefore, when the pump source is in the same output power and the pulse width is $4 \mathrm{~ns}$, there are more pump lines with higher power participate in Raman conversion. This also explains why the second-order Stokes wave is not generated with the same pump power when the pump pulse is $2 \mathrm{~ns}$. 


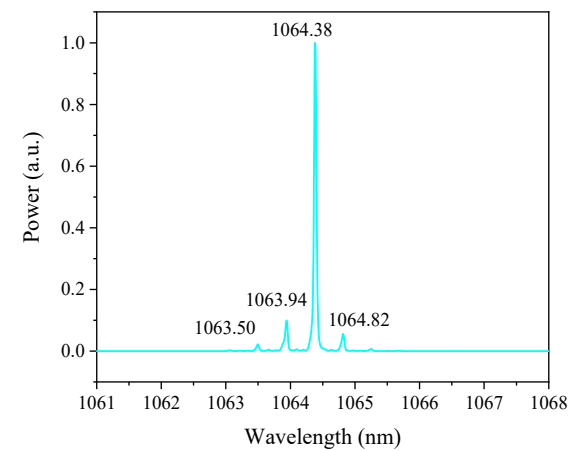

(a)

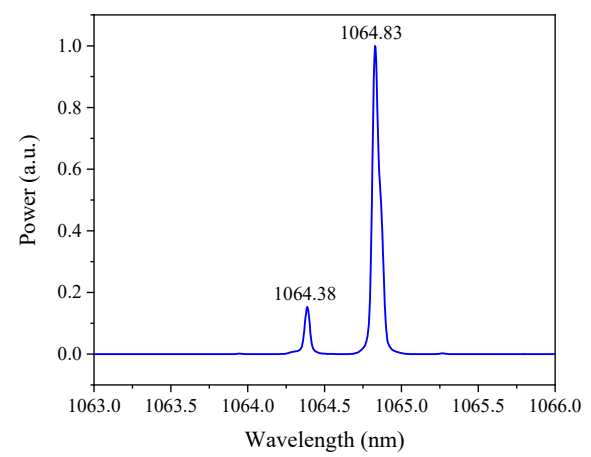

(b)

Figure 6. (a,b) The power spectrums of the pump source at the maximum power with the pulse width of 2 ns and 4 ns, respectively.

The output power characteristics of the FGRL with different pulse width of pump laser are measured, as shown in Figure 7a-c. The solid and hollow patterns in Figure 7a,b represent the Raman power and residual pump power, respectively. Moreover, the coupled pump power is the actual power that transmitted into the gas cavity after the Splice 1. It can be seen that with the increase in the coupled pump power, the Raman power with the pulse width of $2 \mathrm{~ns}$ is detected first. This is because the pump pulse with width $2 \mathrm{~ns}$ is narrower, which means the corresponding peak power is higher than the pulse with the width of $4 \mathrm{~ns}$ when the pump power is the same and exceed the Raman threshold earlier. Both the Raman powers with different pulse widths increase linearly. The residual pump power with the pulse width of $4 \mathrm{~ns}$ drops when the coupled pump power exceeds the Raman threshold, while it keeps increasing when the pulse is 2 ns. It indicates the Raman conversion is not sufficient when the pulse width is $2 \mathrm{~ns}$ in the case. The corresponding Raman efficiency (the ratio of coupled pump power to Raman power in the gas cavity) is calculated, as shown in Figure 7c. The maximum Raman efficiency $28.5 \%$ is obtained with the maximum coupled pump power and the pulse width is $4 \mathrm{~ns}$. Besides, the Stokes pulse energy and the coupled pulse energy are explored in the Figure $7 \mathrm{~d}$. It can be seen that the Stokes energy with the pulse width of $4 \mathrm{~ns}$ is higher in general.

Furthermore, we measured the output power characteristics of the FGRL with different repetition frequencies and the same pulse width of $4 \mathrm{~ns}$. Except for the repetition frequencies, the characteristics of the pump pulse like duration and spectral content are the same. Figure $8 \mathrm{a}, \mathrm{b}$ presents the evolution of Raman power, residual pump power, and Raman efficiency with the increase of coupled pump power, respectively. It can be seen from Figure $8 \mathrm{a}, \mathrm{b}$ that the curves with different repetition frequencies show similar trends. All the Raman powers increase linearly, while all the residual powers increase at first and then drop when the coupled pump power exceed the Raman threshold. Obviously, the optimal repetition frequency is $20 \mathrm{MHz}$ with the lowest Raman threshold and the highest Raman power among the five repetition frequencies. When the repetition frequency is less than $20 \mathrm{MHz}$, the peak powers of the pump pulse and Raman pulse will become higher, which is conducive to the conversion of the second-order Raman laser. When it is higher than $20 \mathrm{MHz}$, less energy of pump pulses that exceed the first-order Raman threshold are converted into Raman pulses. Both situations will reduce the first-order Raman conversion efficiency. Figure $8 \mathrm{c}$ depicts the Raman efficiencies with different repetition frequencies. The maximum Raman efficiency is $28.5 \%$ in $20 \mathrm{MHz}$ as mentioned earlier, which is more efficient than the previous work [14]. The corresponding Raman power is $289 \mathrm{~mW}$, which is the maximum output Raman power. Compared with the previous work [14], the output power is increased by an order of magnitude. The pulse energy of Stokes pulse and coupled pump pulse is explored as shown in Figure 8d. It can be seen when the repetition frequency is $20 \mathrm{MHz}$, the Stokes pulse has the highest energy. 


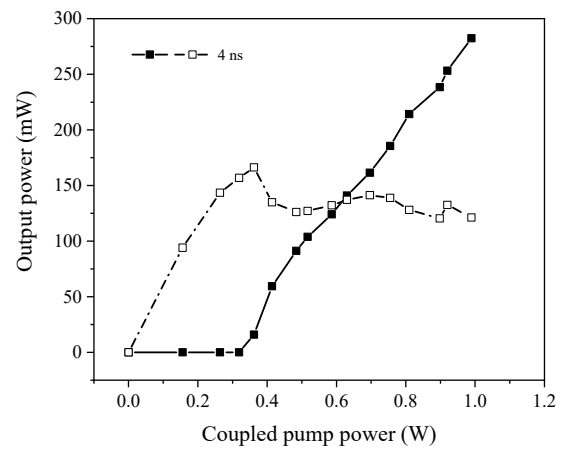

(a)

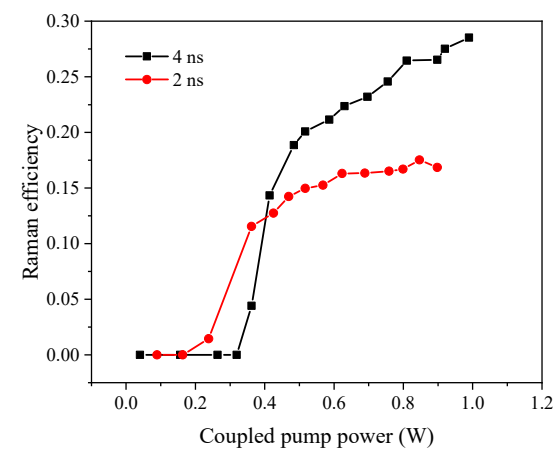

(c)

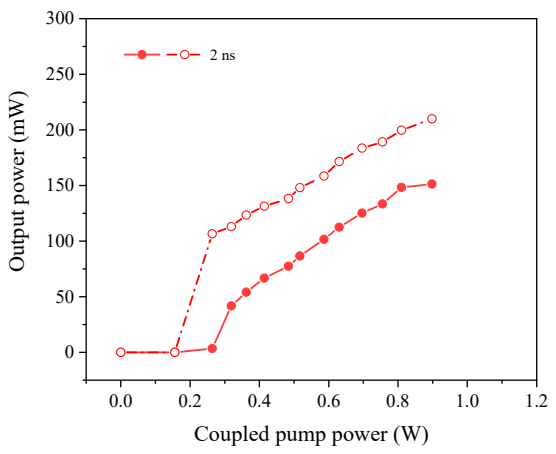

(b)

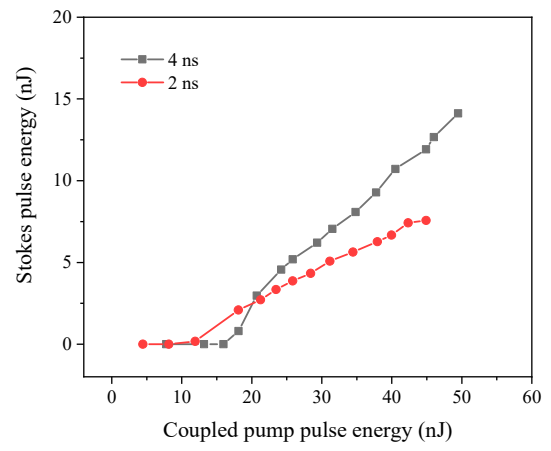

(d)

Figure 7. The output power characteristics of the FGRL with the pulse width of (a) 4 ns and (b) 2 ns. The solid and hollow patterns represent the Raman power and residual pump power, respectively. (c) The Raman efficiency of different pulse widths. (d) The Stokes pulse energy with coupled pump pulse energy in different repetition frequency.

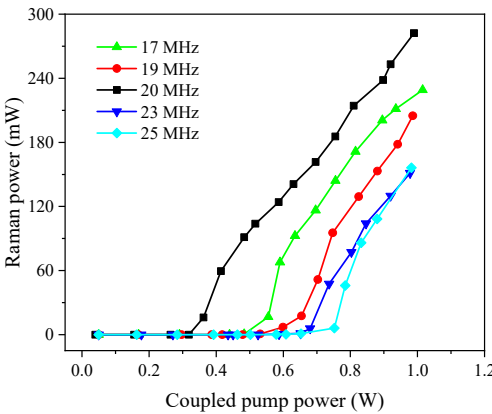

(a)

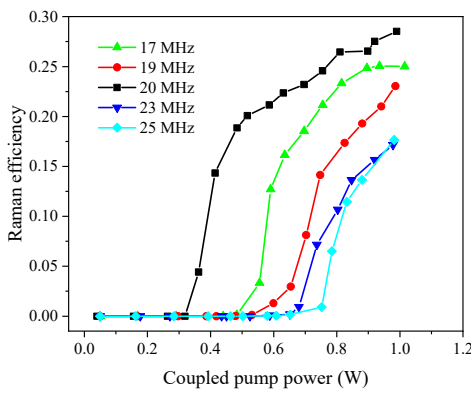

(c)

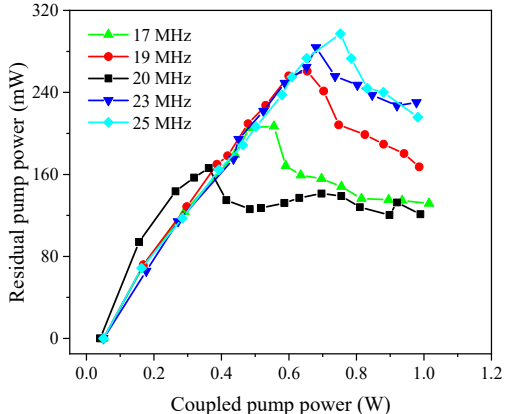

(b)

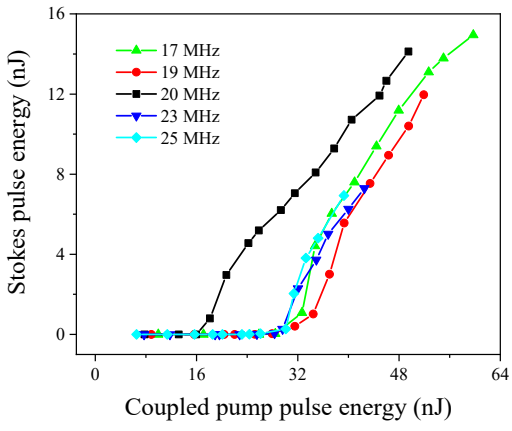

(d)

Figure 8. The output power characteristics of the FGRL with different repetition frequencies (pulse width $4 \mathrm{~ns}$ ). (a-c) The Raman power, residual pump power and Raman efficiency, respectively. (d) The Stokes pulse energy with coupled pump pulse energy in different repetition frequency. 
Figure 9a-d presents the shapes and the series of the pump pulses and the Stokes pulses when the pump power is maximum and the repetition is $20 \mathrm{MHz}$. It can be seen that the frequency of the Stokes pulses is $20 \mathrm{MHz}$, which is the same as the pump pulses. The pulse widths are measured, as shown in Figure 9 a,c. It can be seen that the width of the Stokes pulse is narrower than that of pump pulse. This is not unexpected, because only the center part of the pump pulse that is higher than Raman threshold can be converted into the Stokes pulses.

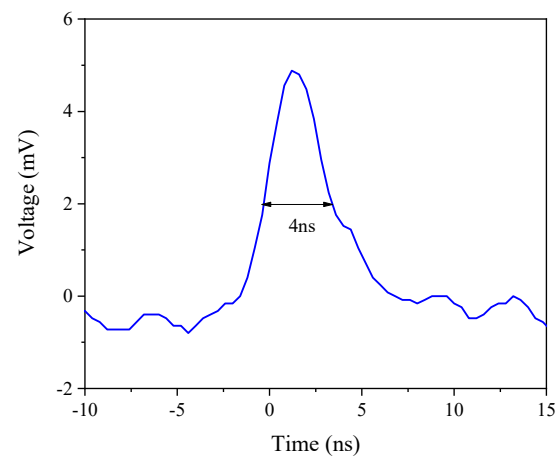

(a)

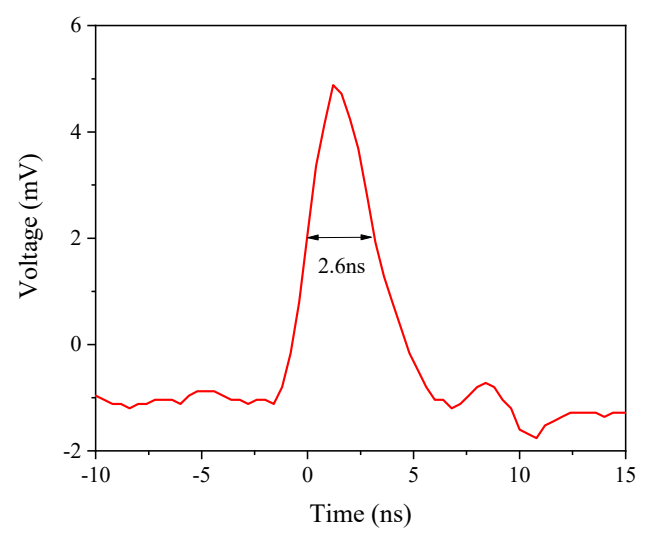

(c)

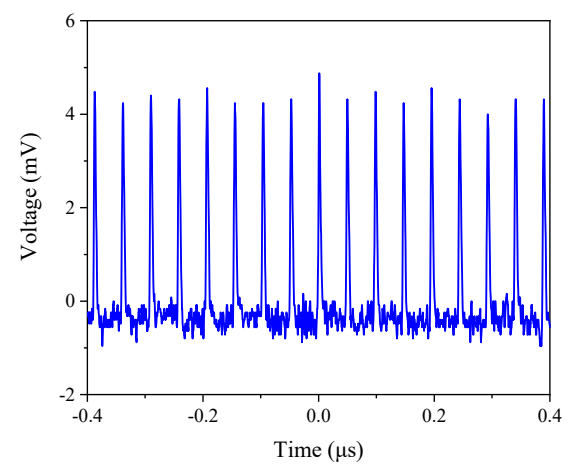

(b)

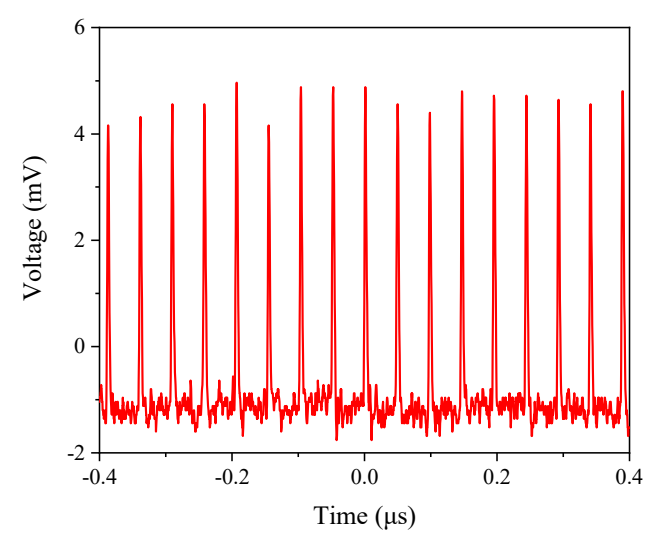

(d)

Figure 9. The temporal characteristic of the FGRL when the pump source is at the maximum power and the repetition is $20 \mathrm{MHz}$. (a,b) The shapes and the series of the pump pulses. (c,d) The shapes and the series of the Stokes pulses.

For the single FGRL in our work, the process of the rotational SRS that generates the first-order and the second-order Stokes waves obey the rules as follows [15]:

$$
\begin{gathered}
\frac{d P_{P}}{d z}=-\frac{\lambda_{S 1}}{\lambda_{P}} g_{S 1} P_{S 1} P_{P}-\alpha_{P} P_{P} \\
\frac{d P_{S 1}}{d z}=g_{S 1} P_{S 1} P_{P}-\alpha_{S 1} P_{S 1}-\frac{\lambda_{S 2}}{\lambda_{S 1}} g_{S 2} P_{S 2} P_{S 1} \\
\frac{d P_{S 2}}{d z}=g_{S 2} P_{S 2} P_{S 1}-\alpha_{S 2} P_{S 2}
\end{gathered}
$$

where the subscripts of $P, S 1$, and $S 2$ represent the pump wave, the first-order Stokes wave, and the second-order Stokes wave, respectively; $z$ is the coordinate of the HC-PCF length; $\lambda$ is the wavelength; $g$ is the steady-state Raman gain coefficient; $P$ is the intensity; and $\alpha$ is the fiber loss. The boundary conditions can be given [11]:

$$
P_{P}(z=0)=\frac{P_{0}}{A_{e f f}}
$$




$$
P_{s 1}(z=0)=\frac{h c \pi \Delta v_{R}}{\lambda_{S 1} A_{e f f}}
$$

where $P_{0}$ is the peak power of the coupled pump pulse in the HC-PCFs; $A_{e f f}$ is the mode field area of the HC-PCFs; $h$ is the Planck constant; $c$ is the light speed $\Delta v_{R}$; is the Raman linewidth. A simple steady-state theoretical model can be established by the formulas above.

In our work, Figure 10a presents the simulation result of the pump pulse shape and the first-order Stokes pulse shape based on the theoretical model. Here $\Delta v_{R}$ is $3.1 \mathrm{GHz}$ [20]. And $g_{S 1}$ is $0.35 \mathrm{~cm} / \mathrm{GW}$ and $g_{S 2}$ is $0.18 \mathrm{~cm} / \mathrm{GW}$, which is estimated and adjusted slightly from in $[10,11,18]$. The mode field diameter of the HC-PCF is $7.5 \mu \mathrm{m}$ and $A_{e f f}$ is $14.06 \pi \mu \mathrm{m}^{2}$. The pump pulse width is $4 \mathrm{~ns}$ and the repetition frequency is $20 \mathrm{MHz}$. It can be seen the Stokes pulse width is $\sim 2.8 \mathrm{~ns}$, which is in good agreement with the experimental results. Figure 10b shows the simulation results of the output power characteristics in the same case. For better comparison, the experimental results are plotted with solid patterns, while the simulation results are plotted with dotted lines. We can find that both the results show the similar trend and with the increase of the coupled pump power, the experimental results are in good agreement with the simulation results at first, then there is a deviation between them. We speculated that the homemade pulsed YDFA may has some continuous wave components which are useless in the Raman conversion and its power increases with the increase of coupled pump power. We hypothesize that by optimizing the performance of the amplifier, we can achieve better agreement between the experimental results and the simulation results.

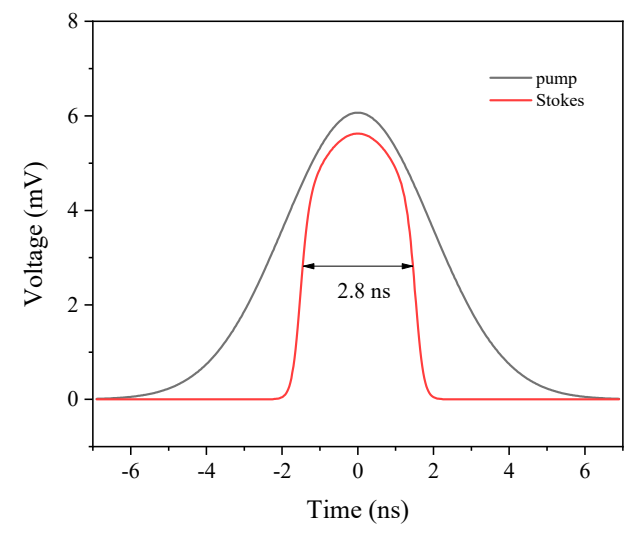

(a)

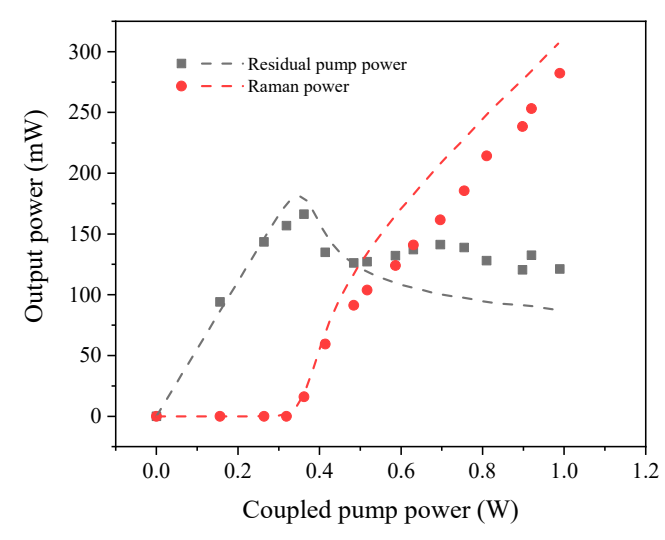

(b)

Figure 10. The simulation results of (a) the pump pulse shape and the first-order Stokes pulse shape and (b) the output power characteristics.

\section{Conclusions}

We have demonstrated the rotational SRS of hydrogen molecules in an all-fiber cavity based on HC-PCFs. The all-fiber gas cavity is fabricated by fusion splicing $49 \mathrm{~m}$ long HC-PCFs filled with 18 bar hydrogen and two sections of solid-core fibers. When pumped by a homemade $1064 \mathrm{~nm}$ YDFA, the pure rotational SRS of hydrogen molecules occurs in the gas cavity. The output characteristics of the FGRL with different repetition frequency and pulse width are explored. The maximum output power with $289 \mathrm{~mW}$ of the first-order Stokes wave at $1135 \mathrm{~nm}$ is obtained and the corresponding Raman efficiency is $28.5 \%$. A steady-state theoretical model is established, and the experimental results are in good agreement with the simulation results. This work makes a further exploration for the applications of the FGRLs. 
Author Contributions: Conceptualization, Z.W. and W.P.; methodology, W.P.; software, W.H. and H.L.; validation, Z.W., H.L. and W.P.; formal analysis, Z.W.; investigation, W.P.; resources, M.W.; data curation, H.L.; writing-original draft preparation, W.P.; writing-review and editing, Z.W.; visualization, W.P. All authors have read and agreed to the published version of the manuscript.

Funding: This work was supported by Outstanding Youth Science Fund Project of Hunan Province Natural Science Foundation (2019JJ20023), National Natural Science Foundation of China (NSFC) (11974427, 12004431) and State Key Laboratory of Pulsed Power Laser Technology (SKL 2020 ZR05).

Institutional Review Board Statement: Not applicable.

Informed Consent Statement: Not applicable.

Data Availability Statement: The data presented in this study are available on request from the corresponding author. The data are not publicly available due to privacy.

Conflicts of Interest: The authors declare no conflict of interest.

\section{References}

1. Minck, R.W.; Terhune, R.W.; Rado, W.G. Laser stimulated Raman effect and resonant four-photon interactions in gases $\mathrm{H}_{2}$, $\mathrm{D}_{2}$, and $\mathrm{CH}_{4}$. Appl. Phys. Lett. 1963, 3, 181-184. [CrossRef]

2. Brink, D.J.; Proch, D. Efficient Tunable Ultraviolet Source Based on Stimulated Raman Scattering of an Excimer-Pumped Dye Laser. Opt. Lett. 1982, 7, 494-496. [CrossRef] [PubMed]

3. Loree, T.R.; Cantrell, C.D.; Barker, D.L. Stimulated Raman emission at $9.2 \mu \mathrm{m}$ from hydrogen gas. Opt. Comm. 1976, 17, 160-162. [CrossRef]

4. Benabid, F. Stimulated Raman Scattering in Hydrogen-Filled Hollow-Core Photonic Crystal Fiber. Science 2002, $298,399-402$. [CrossRef] [PubMed]

5. Benabid, F.; Bouwmans, G.; Knight, J.C.; Russell, P.S.J.; Couny, F. Ultrahigh Efficiency Laser Wavelength Conversion in a Gas-Filled Hollow Core Photonic Crystal Fiber by Pure Stimulated Rotational Raman Scattering in Molecular Hydrogen. Phys. Rev. Lett. 2004, 93, 123903. [CrossRef] [PubMed]

6. Couny, F.; Benabid, F.; Light, P.S. Subwatt Threshold Cw Raman Fiber-Gas Laser Based on $\mathrm{H}_{2}$-Filled Hollow-Core Photonic Crystal Fiber. Phys. Rev. Lett. 2007, 99, 143903. [CrossRef]

7. Wang, Z.; Yu, F.; Wadsworth, W.J.; Knight, J.C. Efficient $1.9 \mu \mathrm{m}$ Emission in $\mathrm{H}_{2}$-Filled Hollow Core Fiber by Pure Stimulated Vibrational Raman Scattering. Laser Phys. Lett. 2014, 11, 105807. [CrossRef]

8. Gladyshev, A.V.; Bufetov, I.A.; Dianov, E.M.; Kosolapov, A.F.; Khudyakov, M.M.; Yatsenko, Y.P.; Kolyadin, A.N.; Krylov, A.A.; Pryamikov, A.D.; Biriukov, A.S.; et al. 2.9, 3.3, and $3.5 \mu \mathrm{m}$ Raman Lasers Based on Revolver Hollow-Core Silica Fiber Filled by ${ }^{1} \mathrm{H}_{2} / \mathrm{D}_{2}$ Gas Mixture. IEEE J. Select. Top. Quantum Electron. 2018, 24, 0903008. [CrossRef]

9. Astapovich, M.S.; Gladyshev, A.V.; Khudyakov, M.M.; Kosolapov, A.F.; Likhachev, M.E.; Bufetov, I.A. Watt-Level Nanosecond $4.42 \mu \mathrm{m}$ Raman Laser Based on Silica Fiber. IEEE Photon. Technol. Lett. 2019, 31, 78-81. [CrossRef]

10. Huang, W.; Li, Z.; Cui, Y.; Zhou, Z.; Wang, Z. Efficient, Watt-Level, Tunable $1.7 \mu \mathrm{m}$ Fiber Raman Laser in $\mathrm{H}_{2}$-Filled Hollow-Core Fibers. Opt. Lett. 2020, 45, 475-478. [CrossRef]

11. Li, H.; Huang, W.; Cui, Y.; Zhou, Z.; Wang, Z. Pure Rotational Stimulated Raman Scattering in $\mathrm{H}_{2}$-Filled Hollow-Core Photonic Crystal Fibers. Opt. Express 2020, 28, 23881-23897. [CrossRef] [PubMed]

12. Thapa, R.; Knabe, K.; Corwin, K.L.; Washburn, B.R. Arc Fusion Splicing of Hollow-Core Photonic Bandgap Fibers for Gas-Filled Fiber Cells. Opt. Express 2006, 14, 9576-9583. [CrossRef] [PubMed]

13. Benabid, F.; Couny, F.; Knight, J.C.; Birks, T.A.; Russell, P.S.J. Compact, Stable and Efficient All-Fibre Gas Cells Using Hollow-Core Photonic Crystal Fibres. Nature 2005, 434, 488-491. [CrossRef]

14. Chen, X.D.; Sun, Q.; Li, H.; Zhao, M.H. Compact All-Fiber Gas Raman Light Source Based on Hydrogen-Filled Hollow-Core Photonic Crystal Fiber Pumped with Single-Mode Q-Switched Fiber Laser. Opt. Fiber Technol. 2013, 19, 486-489. [CrossRef]

15. Li, H.; Pei, W.; Huang, W.; Wang, M.; Wang, Z. Highly Efficient Nanosecond $1.7 \mu \mathrm{m}$ Fiber Gas Raman Laser by $\mathrm{H}_{2}-$ Filled Hollow-Core Photonic Crystal Fibers. Crystals 2020, 11, 32. [CrossRef]

16. Sun, Q.; Mao, Q.; Liu, E.; Rao, R.; Ming, H. Hollow-Core Photonic Crystal Fiber High-Pressure Gas Cell; Sampson, D.D., Ed.; SPIE-International Society of Optical Engineering: Perth, WA, Australia, 2008; p. 700455.

17. Bischel, W.K.; Dyer, M.J. Wavelength Dependence of the Absolute Raman Gain Coefficient for the Q(1) Transition in $\mathrm{H}_{2}$. J. Opt. Soc. Am. B 1986, 3, 677-682. [CrossRef]

18. Xiao, L.; Demokan, M.S.; Jin, W.; Wang, Y.; Zhao, C.-L. Fusion Splicing Photonic Crystal Fibers and Conventional Single-Mode Fibers: Microhole Collapse Effect. J. Lightwave Technol. 2007, 25, 3563-3574. [CrossRef]

19. Hanson, F.; Poirier, P. Stimulated Rotational Raman Conversion in $\mathrm{H}_{2}, \mathrm{D}_{2}$, and HD. IEEE J. Quantum Electron. 1993, 29, 2342-2345. [CrossRef]

20. Herring, G.C.; Dyer, M.J.; Bischel, W.K. Temperature and Density Dependence of the Linewidths and Line Shifts of the Rotational Raman Lines in $\mathrm{N}_{2}$ and $\mathrm{H}_{2}$. Phys. Rev. A 1986, 34, 1944-1951. [CrossRef] [PubMed] 